\title{
Fatores intervenientes à prática do mountain bike junto à natureza
}

\author{
Factors involved in the practice of \\ mountain biking in nature
}

\author{
Jairo Antônio da PAIXÃO' \\ Marizabel KOWALSKI ${ }^{2}$
}

\begin{abstract}
Resumo
Este estudo objetivou analisar fatores motivacionais ligados à adesão e permanência na modalidade mountain bike de um grupo de 29 praticantes, entre amadores e profissionais, com média de idade de 30 anos (considerou-se o desvio-padrão com significância $<0,05 \%)$. A partir da aplicação de entrevista semiestruturada e da técnica de análise do discurso dos dados coletados, foi possível constatar que os fatores motivacionais que mais influenciam na adesão e permanência pelos praticantes no mountain bike são de natureza intrínseca; além da busca pelo risco, da aventura e de fortes emoções, inclui-se a possibilidade da integração saúde e natureza.
\end{abstract}

Unitermos: Fatores de risco; Meio ambiente; Natureza; Satisfação pessoal.

\begin{abstract}
The aim of this study was to investigate the motivational factors related to adherence and permanence in mountain biking. The subjects were 29 amateur and professional practitioners with a mean age of 30 years. The data analysis considered the standard deviation, with a significance level $<0.05 \%$. Using the semi-structured interview technique to collect the data, with discourse analysis, it was possible to affirm that the motivational factors that influence adherence and permanence in mountain biking practitioners are intrinsic factors. These factors go beyond the search for risk, adventure and thrills, including the possibility of integrating health and nature.
\end{abstract}

Uniterms: Risk factors; Environment; Nature; Pessoal satisfaction.

As escolhas que fazemos no decorrer da vida tomam parte no somatório de fatores acionados por objetivos e metas que buscamos realizar em determinados momentos. Envolvem todo e qualquer indi- víduo e, por sua vez, perpassam as diferentes dimensões que compõem a vida em sociedade como, por exemplo, as sociais, culturais e econômicas. A predisposição desses fatores incorpora elementos intrínsecos e

1 Universidade Federal de Ouro Preto, Centro Desportivo, Laboratório de Estudos Pedagógicos em Educação Física. R. Diogo de Vasconcelos, 122, Campus Universitário, Morro do Cruzeiro, s/n., 35400-000, Ouro Preto, MG, Brasil. Correspondência para/Correspondence to: J.A. PAIXÃO. E-mail: <jairopaixao2004@yahoo.com.br>.

2 Universidade Federal de Viçosa, Centro de Ciências Biológicas e da Saúde, Departamento de Educação Física. Viçosa, MG, Brasil. Apoio: Conselho Nacional de Desenvolvimento Científico e Tecnológico e da Fundação de Amparo à Pesquisa do Estado de Minas Gerais. 
extrínsecos fundamentais para a efetivação de ações que, uma vez empreendidas pelas pessoas, podem vir a propiciar mudanças significativas nas trajetórias, tanto individuais quanto coletivas, em uma dada comunidade.

Mudanças desta ordem envolvem diferentes aspectos motivacionais que se destacam como elementos impulsionadores do desenvolvimento humano cognitivo e motor, como, por exemplo, a emoção (Horn, 2002). O elemento cognitivo relacionado à democratização da informação e ao desenvolvimento individual e que, por sua vez, impulsiona o indivíduo na busca de alternativas qualitativas na vida cotidiana, diferencia o ser humano de outros seres vivos (Drake \& Miller, 1969). O que se pretende justificar é que a ação motora é o ato motivacional que revela o envolvimento emocional-afetivo do indivíduo, ou seja, o elemento motor é significativo para a formulação da busca subjetiva, transformando em ação e motivando o indivíduo para a realização de uma meta singular para ele e significativa para a coletividade.

Nessa perspectiva, pode-se entender por motivação um conjunto de forças intra e interdirecionais de comportamentos atitudinais do ser humano, objetivando atingir metas que, a priori, o levem ao melhoramento da qualidade de vida e, a posteriori, que expressem mudanças significativas na comunidade (Cid, 2002). No sentido etimológico, a palavra motivação expressa a ideia de "mover." Percebe-se, nesse sentido, que a motivação conduz o comportamento do indivíduo para um determinado incentivo no qual ele poderá vivenciar momentos de prazer ou, ainda, de alívio de uma situação desagradável (Atkinson, 2002).

Os motivos que levam o indivíduo a realizar ações podem se manifestar de forma intrínseca ou extrínseca (Tresca \& De Rose, 2000). Os motivos intrínsecos resultam da vontade própria do indivíduo e podem estar relacionados às necessidades pessoais de afirmação em um determinado grupo social, esportivo ou familiar, sucesso pessoal e profissional, dentre outros (Cratty, 1984; Tresca \& De Rose, 2000). Já os motivos extrínsecos estão segmentados aos fatores externos e atingíveis por meio de tarefas e, acima de tudo, são tidos como recompensáveis por sinais positivos e/ou negativos socialmente reconhecíveis (Cratty, 1984; Horn, 2002; Serpa, 1992; Tresca \& De Rose, 2000).
Na área social, com a constante influência dos indivíduos sobre o ambiente, é compreensível que o desenvolvimento da emotividade capacite as pessoas a maximizarem os benefícios desta permuta ininterrupta de influências no relacionamento indivíduo-meio.

Dentre as inúmeras manifestações humanas, aquelas ligadas ao âmbito desportivo propiciam o desdobramento de aspectos relacionados às vivências de emoções. Nesse sentido, as práticas esportivas preenchem importantes funções sociais, que, segundo Proni e Lucena (2002), são comumente categorizadas em funções manifestas, latentes ou agonísticas. Essas funções dizem respeito à supressão das necessidades de atividade física, à compensação do estresse provocado pela rotina de trabalho e à satisfação dos instintos mais profundos do ser humano, como o instinto combativo, que está relacionado à luta pela sobrevivência e ao impulso sexual, respectivamente. Em outras palavras, são espaços destinados a excessos e euforias coletivas, conclamados a inverter e alterar os tabus do mundo cotidiano e colocá-los literalmente do avesso. São lugares de "descontrolecontrolado".

Nessa perspectiva, ao se considerar as especificidades inerentes às modalidades que compõem o esporte de aventura no meio natural (risco controlado, vertigem, superação de limites, adrenalina e fortes emoções), os fatores motivacionais ganham destaque e se apresentam subjacentes a questões como essas: "O que faz alguém sair de seu mundo seguro e cômodo e buscar o que se encontra fora das regras, transpondo seus limites para buscar aquilo que considera sagrado?" "Quais as razões que levam pessoas de nível socioeconômico relativamente alto e de prestígio social a deixarem a vida urbana para se lançar em uma aventura de vida simples, que exige audácia e ousadia, atenção constante e muitas restrições?". "O que leva as pessoas a adentrarem a mata, escalar montanhas ou descer rapel em cachoeiras?". "Que fascinação exercem essas atividades sobre seus seguidores, impulsionando-os a dedicar grande parte de seu tempo e de suas vidas a comprovar seus limites?" (C. Costa, 2006).

O surgimento ininterrupto de novas modalidades, acompanhado pelo crescente número de adeptos de diferentes formações culturais, faixas etárias, níveis sociais e campos de atuação profissional, fornece 
pistas para se entender o esporte de aventura como uma realidade no âmbito atual e que, por sua vez, apresenta inúmeras possibilidades de adesão e prática (Marinho \& Bruhns, 2003).

Nesse sentido, a teoria do flow-feeling (experiência do fluir, da fluidez) desenvolvida por Csikszentimihalyi (1975) contribui para o entendimento dessas contradições que permeiam a opção pela prática das diferentes modalidades que compõem o esporte de aventura pelo indivíduo na contemporaneidade. Originalmente, esta teoria foi desenvolvida para explicar a popularidade de certas atividades físicas que apresentam características como desgaste, risco e certo grau de dificuldade, e que exigem do indivíduo considerável persistência (Cater, 2006). A experiência do fluir caracteriza-se pelo completo envolvimento do indivíduo na realização de uma atividade física que, por sua vez, tenha sido escolhida de forma espontânea; permite detectar importantes indícios de como praticantes de mountain bike, por exemplo, mantêm certo nível de motivação para iniciar e manter uma prática regular desta atividade outdoor (Cater, 2006).

Nesta abordagem, Marinho (2007) salienta que a procura pelas práticas físicas na natureza ocorrem, muitas vezes, pelo desejo de se experimentar o novo, pela busca de emoções prazerosas e novas significações na relação do indivíduo junto ao meio natural. Reforçando e completando esta ideia, Cid (2002) afirma que a escolha de determinada prática física não se dá de forma aleatória: por trás de uma escolha está algo ou alguém que, consciente ou inconscientemente, exerceu influências para aquela prática física.

Geralmente, o interesse pela adoção de uma modalidade esportiva associa-se à forma como o indivíduo ocupa o seu tempo livre ou, ainda, vem associado à saúde e ao bem-estar, em resposta às necessidades individuais e sociais (Nunomura, 1998). Tais necessidades podem variar de acordo com idade, sexo, influências da mídia, além de fatores sociais, econômicos e culturais (Machado, 2006; Nunomura, 1998; Santos \& Knijnik, 2006). Somado a tudo isso, encontram-se as necessidades subjetivas do homem, o espírito aventureiro e o desejo de aventurar-se (Marinho, 2007).

Como atividade esportiva outdoor, o mountain bike caracteriza-se como uma modalidade esportiva na qual o praticante perfaz um trajeto no meio natural em uma bicicleta tendo que vencer obstáculos em terrenos irregulares e acidentados. Desta forma, como as diferentes modalidades que compõem o esporte de aventura, o mountain bike apresenta o fator risco como uma constante em sua prática, tanto na perspectiva da competição quanto do lazer, exigindo de seus praticantes variadas adaptações psicológicas, como a capacidade de controlar suas emoções diante do risco eminente frente às disposições geográficas, cenários naturais, adversidade climática, bem como dos esforços físicos, e a busca pela superação de limites (Cater, 2006; C. Costa, Diniz \& Pereira, 1988; V. L. M. Costa, 2006; Cratty, 1984).

Segundo Gaulrapp, Weber e Rosemeyer (2001), dentre os principais fatores de risco na referida modalidade encontram-se as pistas escorregadias, falta de conhecimento do trajeto a ser percorrido e velocidade excessiva.

Procedendo a uma análise do assunto, este estudo buscou analisar fatores motivacionais intervenientes à aderência e permanência no mountain bike, tendo em vista especificidades como risco, emoção, sensação de adrenalina e ambiente natural que permeiam a prática de esporte de aventura e risco calculado na natureza.

\section{Método}

O estudo caracterizou-se como descritivo-exploratório (Drake \& Miller, 1969; Malhotra, 2000; Mattos, Rosseto \& Blecher, 2004), que tem como características observar, registrar, analisar, descrever e correlacionar fatos ou fenômenos sem manipulá-los, procurando descobrir com precisão a frequência em que o fenômeno ocorre e sua relação com outros fatores. No processo de construção e validação do instrumento de coleta de dados, foi empregada a técnica Delphi (Duffield, 1993; Thomas \& Nelson, 2002) a partir de uma sucessão de pareceres de três professores doutores (especialistas na área), perfazendo um total de quatro fases, obtendo-se consenso sobre as questões investigativas sobre os fatores motivacionais ligados à adesão à prática da modalidade mountain bike.

Como instrumento de coleta de dados foi utilizada entrevista semiestruturada, que, nas palavras de Negrine (2004), possibilita a aquisição de informações 
relevantes para o estudo, uma vez que permite ao entrevistado discorrer sobre aspectos que acredita serem importantes sobre o tema de estudo em questão.

Empregou-se a análise interpretativa nos dados coletados que, segundo Triviños (1995), apoia-se em três aspectos fundamentais: (1) nos resultados encontrados no estudo e nas respostas dos instrumentos; (2) na fundamentação teórica e manejo dos conceitos-chave das teorias e de outros pontos de vista, e (3) na experiência pessoal do investigador.

A amostra foi constituída por 29 indivíduos, que atenderam aos critérios de inclusão a partir de um grupo de 38 praticantes de mountain bike, sendo 23 do sexo masculino e seis do sexo feminino, com idade variando entre 19 e 41 anos, na cidade de Governador Valadares (MG). Os critérios de inclusão foram: indivíduos maiores de 18 anos, sexo masculino ou feminino, que praticavam a modalidade do mountain bike em áreas naturais da cidade de Governador Valadares, e assinatura do Termo de Consentimento Livre e Esclarecido (TCLE). Os critérios de exclusão foram recusa da assinatura do TCLE, idade inferior a 18 anos, bem como o não interesse em participar da investigação.

Dentre os procedimentos necessários para a realização das entrevistas junto aos sujeitos do estudo fez-se necessária uma prévia marcação do dia e horário dos encontros. Estes se deram de forma individual em locais de trabalho, bem como nas residências e locais públicos. Procurou-se explicar aos entrevistados acerca do estudo, seu propósito, deixando-os, assim, a par da relevância de sua contribuição para a pesquisa. Os eixos norteadores da entrevista foram: motivo de início da prática; motivação para a manutenção; frequência com que pratica; dificuldades para a prática e conhecimento dos benefícios (Nunomura, 1998; Saba, 2001; Santos \& Knijnik, 2006).

Para análise dos dados foi utilizada a técnica de análise de conteúdo, que se refere a um conjunto de técnicas que visa a obter, por procedimentos sistemáticos e objetivos de descrição do conteúdo das mensagens, indicadores quantitativos ou não que permitam a inferência de conhecimentos relativos às condições de produção/recepção (variáveis inferidas) dessas mensagens (Bardin, 2006). Após a análise interpretativa dos dados, os mesmos foram categorizados e quantificados a partir da frequência de ocorrência. As análises estatísticas foram realizadas por meio de estatística descritiva, utilizando-se os procedimentos de média e desvio-padrão.

\section{Resultados}

De acordo com a Tabela 1, dentre os motivos que mais influenciaram a adesão à modalidade do mountain bike ressalta-se a familiaridade do praticante com o ato de pedalar. Em seguida, em uma ordem decrescente de frequência, tem-se a influência de amigos e familiares que praticam a modalidade em questão.

Dentre os fatores intervenientes à aderência da modalidade, os resultados mostrados na Tabela 2 evidenciam uma profusão de motivos, como: socialização; preocupação com a forma física (estética); forma de se livrar do estresse; busca de sensações, como aquela que se sente com a liberação de adrenalina; fluidez; fortes emoções e, ainda, o bem-estar propiciado pelo ambiente natural.

\section{Tabela1}

Motivos de início da prática do mountain bike. Governador Valadares (MG), 2010

\begin{tabular}{lcc}
\hline Categorias & F & $\%$ \\
\hline Familiaridade com bicicleta & 13 & 45 \\
Influência da mídia & 9 & 31 \\
Influência de familiares e amigos & 7 & 24 \\
\hline Total & 29 & 100 \\
\hline
\end{tabular}

\section{Tabela 2}

Fatores de aderência ao mountain bike. Governador Valadares (MG), 2010

\begin{tabular}{lcc}
\hline Categorias & F & $\%$ \\
\hline Socialização/estabelecer novas amizades & 9 & 31 \\
Maneira prazerosa de manter a forma física & 7 & 24 \\
Forma de se livrar do estresse do cotidiano & 7 & 24 \\
Sensação de liberdade/adrenalina/fluidez & 4 & 12 \\
Contato com diferentes paisagens naturais & 3 & 9 \\
\hline Total & 29 & 100 \\
\hline
\end{tabular}


A Tabela 3 mostra os resultados referentes à frequência com que os sujeitos entrevistados praticam a modalidade. Observa-se que essa frequência ocorre com certo predomínio nos finais de semana.

A Tabela 4 apresenta os resultados referentes às maiores dificuldades enfrentadas pelos sujeitos do estudo para a prática do mountain bike. O desrespeito no trânsito por parte dos motoristas surge com destaque. Em seguida, foram apontados o descaso dos órgãos públicos com os locais naturais de prática da modalidade em questão e, ainda, a aquisição dos equipamentos necessários para a prática do mountain bike.

Os praticantes, devido à faixa etária e preferência, não buscam a competitividade própria dos jogos e modalidades esportivas no âmbito profissional ou de

\section{Tabela 3}

Frequência com que pratica o mountain bike. Governador Valadares (MG), 2010

\begin{tabular}{lcc}
\hline Categorias & $F$ & $\%$ \\
\hline Nos finais de semana & 19 & 67 \\
Esporadicamente & 6 & 21 \\
Até duas vezes por semana & 3 & 9 \\
Até três vezes por semana & 1 & 4 \\
\hline Total & 29 & 100 \\
\hline
\end{tabular}

\section{Tabela 4}

Dificuldades para a prática do mountain bike. Governador Valadares (MG), 2010

\begin{tabular}{lcc}
\hline Categorias & F & $\%$ \\
\hline Desrespeito no trânsito & 15 & 52 \\
Descaso dos órgãos públicos com o meio natural & 7 & 24 \\
Aquisição e manutenção dos equipamentos & 5 & 17 \\
Dificuldade de patrocínio & 2 & 7 \\
\hline Total & 29 & 100 \\
\hline
\end{tabular}

\section{Tabela 5}

Conhecimento dos benefícios do mountain bike. Governador Valadares (MG), 2010

\begin{tabular}{lcc}
\hline Categorias & $F$ & $\%$ \\
\hline Condicionamento físico-motor & 16 & 57 \\
Contato com a natureza & 9 & 31 \\
Aumentar o círculo de amizades & 4 & 12 \\
\hline Total & 29 & 100 \\
\hline
\end{tabular}

rendimento. Predominam os objetivos listados na Tabela 5 .

Observa-se que, dentre os principais objetivos que os praticantes buscam atingir na prática do mountain bike, destacam-se o condicionamento físico-motor, o contato com a natureza e as relações de amizade entre pessoas que fazem parte do grupo de praticantes.

\section{Discussão}

A familiaridade com equipamentos, atividades afins e, sobretudo, o domínio de habilidades motoras específicas de uma determinada modalidade esportiva podem configurar elementos decisivos para a aderência e desempenho de praticantes de mountain bike nesta atividade (Paixão, Gabriel, Tucher, Kowalski \& Menezes Costa, 2011). Aliada à familiaridade com o ato de pedalar, foi apontada ainda a influência midiática, que vem contribuindo de forma decisiva para com a difusão das mais variadas modalidades esportivas na contemporaneidade, como destacam Figueira e Goellner (2005). Juntamente com as atividades físicas são agregados outros termos, como saúde, beleza, performance, longevidade, juventude, dentre outras possibilidades, cuja combinação é promissora de felicidade, consumo e qualidade de vida. Esta situação contribui sobremaneira para que as pessoas possam ter acesso a uma dada modalidade esportiva de forma mais rápida e fácil.

Percebe-se que familiares e amigos podem exercer significativa influência, do ponto de vista positivo ou negativo, para a participação do indivíduo em esportes e atividades físicas. A relevância da influência de familiares e adultos na aderência a uma dada modalidade esportiva é tanta que esta tem sido uma temática amplamente discutida pela psicologia do esporte (Becker, 2000; De Rose, 2002; Weinberg \& Gould, 2001).

O surgimento das diferentes modalidades de esporte de aventura, ênfase aqui à modalidade do mountain bike, dá-se em decorrência de uma série de fatores no contexto social, como a racionalização do tempo, configuração atual do trabalho, necessidade de expressão e mudanças no ser humano, (re)encontro com o meio natural, prática física, contemplação, superação dos próprios limites, lazer, possibilidade de experimentar fortes emoções, prazer, liberdade, promoção da saúde e qualidade de vida. 
Vive-se atualmente uma fase de complexa mudança, com perdas de valores e estilos de vida, vazio existencial e preocupações permanentes. Busca-se "algo" desconhecido e indefinido; daí o interesse cada vez maior pelas modalidades de esporte de aventura (Marinho, 2007). Termos como adrenalina, fortes emoções e superação passaram a fazer parte da linguagem cotidiana daqueles que se entregam à aventura e aos momentos em que experimentam as fortes emoções características dessas atividades. Desta forma, os fatores intervenientes à aderência da modalidade, apresentados na Tabela 2, identificam-se com aqueles relacionados à motivação intrínseca, que são mais duradouros e persistentes e permeiam a própria prática, bem como os sentimentos que ela provoca nos praticantes (Cid, 2002). Trata-se de motivos internos ao praticante, como a alegria, sensação de realização, dentre outros, que promovem o desenvolvimento de outros tipos de necessidades, tais como a competência e a autonomia humana (C. Costa et al., 1988).

A frequência com que é praticado o mountain bike, em quase a totalidade do grupo analisado, fornece pistas para pensar nesta como uma atividade que ocorre na perspectiva do lazer. Essas informações, somadas à informação sobre a faixa etária dos sujeitos que fizeram parte do estudo (19 a 41 anos de idade, com exceção de um praticante) não denotam treinamento/preparação visando a competições; a motivação para se praticar esta modalidade esportiva debruça-se sobre três questões fundamentais: saber o que leva um sujeito a iniciar determinada atividade, a razão porque privilegia determinado objetivo e a causa pela qual persiste em realizar esse objetivo (Cid, 2002; C. Costa et al., 1988).

É preciso considerar a realidade das cidades em que existem áreas naturais de prática de modalidades de esporte de aventura. De acordo com a observação e mesmo os depoimentos dos entrevistados, vê-se que a grande maioria dos praticantes desloca-se para o ambiente de prática em suas próprias bicicletas, perfazendo, assim, consideráveis trechos de rodovias, vias e ruas. Enfim, esta situação coloca esses praticantes diante de riscos e incertezas que permeiam o trânsito, o que pode incidir em stress e possibilidades de acidentes em rodovias.
Quanto à dificuldade de aquisição e manutenção de equipamentos para a prática do mountain bike, assim como para outras modalidades que não sejam aquelas ligadas aos esportes tradicionais, observa-se que, por se tratar de cidades de pequeno porte, o acesso aos equipamentos fica mais restrito.

É notória a estreita relação entre as modalidades que compõem o esporte de aventura e natureza e sua interação com o praticante. No entanto, como se percebe neste estudo, o ambiente não se limita somente a um cenário para a realização das modalidades do referido segmento esportivo: evidenciou-se certa preocupação por parte dos praticantes com os órgãos públicos e sua responsabilidade para com o ambiente natural.

Nota-se que os depoimentos dos praticantes sobre os objetivos e/ou benefícios proporcionados pela prática do mountain bike - por exemplo, o contato com a natureza, o desenvolvimento de capacidades saudáveis tais como o condicionamento físico-motor, momento de lazer e convivência social -, coadunam-se com os resultados encontrados em estudo realizado por White, Waskey, Brodehl e Foti (2006).

Há que se acrescentar que os grupos humanos que se formam em torno da prática do mountain bike, que buscam e compartilham vivências instigantes junto à natureza, possibilitam, na mesma proporção, o estabelecimento e estreitamento de laços de amizade entre seus pares. Maffesoli (2006) observa um movimento que busca estabelecer resistência a esse quadro na sociedade pós-moderna. Para isso, o autor utiliza-se da expressão metafórica "tribos urbanas" para designar grupamentos humanos que buscam, acima de tudo, contestar o individualismo e outras implicações percebidas nas relações sociais que se estabelecem na contemporaneidade.

Ao se considerar o sentido atribuído pelo referido autor à expressão "tribo urbana" na sociedade contemporânea, é possível estabelecer uma espécie de analogia entre os integrantes dessas tribos e os praticantes de mountain bike. Os integrantes associam-se objetivando compartilhar riscos, fortes emoções, benefícios físicos e outras sensações proporcionadas pela referida modalidade esportiva como um meio de expressar-se como humanos pertencentes a um dado grupo na sociedade. 
No que se refere aos motivos que influenciam a adesão e a permanência na prática do mountain bike, verificou-se que, independentemente da variável gênero, sobressaíram-se aqueles intrínsecos. De acordo com o estilo do grupo analisado, os objetivos e aspirações com a modalidade em questão confirmaram o que mostra a literatura consultada. Outro fato destacado pelo estudo foi a existência de sujeitos que aderem à prática de uma modalidade esportiva - no caso, o mountain bike - priorizando aspectos diferentes daqueles ligados à performance: buscam uma profusão de objetivos e benefícios que permeiam a vida.

Por fim, vislumbram-se possibilidades de lançar olhares positivos em relação à interação do homem com a natureza por meio da prática do mountain bike; para tanto, é desejável e aconselhável que sejam fomentadas leis municipais no sentido de não somente estruturar, mas preservar os ambientes naturais de prática de modalidades de esporte de aventura. Tal esforço poderá se efetivar mediante a implementação de placas sinalizadoras, campanhas de conscientização para a preservação da natureza para a população local e instalação de recipientes para coleta de lixo reciclável no local de prática, tanto do mountain bike quanto de outras modalidades de esporte de aventura que o relevo da região propicie.

\section{Referências}

Atkinson, R. C. (2002). Introdução à psicologia de Hilgard (13a ed.). Porto Alegre: Artmed.

Bardin, L. (2006). Análise de conteúdo. Lisboa: Edições 70.

Becker, J. (2000). Psicologia aplicada a criança no esporte. Novo Hamburgo: Edelbra.

Cater, C. I. (2006). Playing with risk? Participant perceptions of risk and management implications in adventure tourism. Tourism Management, 27(2), 317-325.

Cid, L. F. (2002). Alteração dos motivos para a prática desportiva das crianças e jovens. Lecturas, Educación Física y Deportes, Revista Digital, 8(55). Recuperado en jul. 10, 2008, Disponivel en <http://www.efdeportes.com>.

Costa, C., Diniz, J., \& Pereira, P. (1988). A motivação dos alunos para a educação física: a sua influência no comportamento nas aulas. Revista Horizonte, 86, 7-15.

Costa, V. L. M. (2006). Práticas corporais na natureza: tendências da pós-modernidade (Aula inaugural não-publicada). Programa de Doutorado em Ciência do Desporto, Universidade de Trás-os-Montes e Alto D'ouro, Vila Real, Portugal.
Cratty, B. J. (1984). Psicologia do esporte. Rio de Janeiro: Prentice Hall.

Csikszentmihalyi, M. (1975). Beyound boredom and anxiety. San Francisco: Jossey-Bass.

De Rose, J. D. (2002). Esporte e atividade física na infância e adolescência: uma abordagem multidisciplinar. Porto Alegre: Artmed.

Drake, J. E., \& Miller F. J. (1969). Marketing research: Intelligence and management. Stranton, PA: International Textbooks.

Duffield, C. (1993). The Delphi Technique: A comparison of results obtained using two expert panels. International Journal of Nursing Studies, 30(3), 277-37.

Figueira, M. L. M., \& Goellner, S. V. (2005). A promoção do estilo atlético na revista capricho e a produção de uma representação de corpo adolescente feminino contemporâneo. Revista Brasileira de Ciências do Esporte, 26(2), 35-48.

Gaulrapp, H., Weber, A., \& Rosemeyer, B. (2001). Injuries in mountain biking. Knee Surgery, Sports Traumatology, Arthroscopy, 9(1), 48-53.

Horn, T. S. (2002). Advances in sport psychology. Champaign: Human Kinetics.

Machado, F. H. (2006). Mundo emocionado e as atividades físicas de aventura na natureza. In G. M. Schwartz (Ed.), Aventuras na natureza: consolidando significados (pp.103-123). Jundiaí: Fontoura.

Maffesoli, M. (2006). Os tempos das tribos: o declínio do individualismo nas sociedades de massa (4ª ed.). Rio de Janeiro: Forense.

Malhotra, N. K. (2000). Pesquisa de marketing: uma orientação aplicada. Porto Alegre: Bookman.

Marinho, A. (2007). Lazer, natureza e aventura: compartilhando emoções e compromissos. In A. P. C. Almeida \& L. P. Costa (Eds.), Meio ambiente, esporte, lazer e turismo (pp.331-319). Rio de Janeiro: Gama Filho.

Marinho, A., \& Bruhns, H. T. (2003). Turismo, lazer e natureza. São Paulo: Manole.

Mattos, M. G, Rosseto, A. J. J. R., \& Blecher, S. (2004). Teoria e prática da metodologia da pesquisa em educação física: construindo sua monografia, artigo científico e projeto de ação. São Paulo: Phorte.

Negrine, A. (2004). Instrumentos de coleta de informações na pesquisa qualitativa. In V. N. Molina \& A. N. S. Triviños (Eds.), A pesquisa qualitativa na educação física (pp.196-212). Porto Alegre: Sulina.

Nunomura, M. (1998). Motivos de adesão à atividade física em função das variáveis idade, sexo, grau de instrução e tempo de permanência. Revista Brasileira de Atividade Física e Saúde, 3(3), 45-58.

Paixão, J. A., Gabriel, R. E. C. D., Tucher, G., Kowalski, M., \& de Menezes Costa, V. L. (2011). Risco e aventura no esporte na percepção do instrutor. Psicologia \& Sociedade, 23(2), 415-425.

Proni, M., \& Lucena, R. F. (2002). Esporte: história e sociedade. Campinas: Editores Associados. 
Saba, F. K. F. (2001). Aderência à prática do exercício físico em academias. São Paulo: Manole.

Santos, S. C., \& Knijnik, J. D. (2006). Motivos de adesão à prática de atividade física na vida adulta intermediária. Revista Mackenzie de Educação Física e Esporte, 5(1), 23-34.

Serpa, S. (1992). Motivação para a prática desportiva: validação preliminar do questionário de motivação para as actividades desportivas. In F. Sobral \& A. Marques (Eds.), FACDEX: desenvolvimento somato-motor e factores de excelência desportiva na população escolar portuguesa (Vol.2, pp.89-97). Lisboa: Ministério da Educação.

Thomas, J. R., \& Nelson, J. K. (2002). Métodos de pesquisa em atividade física (3a ed.). Porto Alegre: Artmed.

Tresca, R. P., \& De Rose J. D. (2000). Estudo comparativo da motivação intrínseca em escolares praticantes e não praticantes de dança. Revista Brasileira de Ciência e Movimento, 8(1), 9-13.

Triviños, A. N. S. (1995). Introdução à pesquisa em ciências sociais: a pesquisa qualitativa em educação. São Paulo: Atlas.

Weinberg, R. S., \& Gould, D. (2001). Fundamentos dapsicologia do esporte e do exercício. Porto Alegre: Artmed.

White, D. D., Waskey, M. T., Brodehl, G. P., \& Foti, P. E. (2006). A comparative study of impacts to mountain bike trails in five common ecological regions of the southwestern US. Journal of Park and Recreation Administration, 24(2), 21-41.

Recebido em: 5/5/2010

Versão final em: 14/3/2012

Aprovado em: 16/4/2012 